\title{
Lujo tópico y distópico: Un marco para el diseño y la publicidad
}

\author{
JAVIER GONZÁLEZ SOLAS ${ }^{1}$ \\ Universidad Complutense de Madrid
}

He intentado mostrar cómo la manipulación de la emulación social ha hecho que el hombre persiga «lujos» donde previamente ha comprado «decoro», y «decoro» donde previamente compró sólo «necesidades» ${ }^{2}$.

MCKendRICK, N., BREWER, J. and Plumb, J. H.

El sistema de necesidades es producto del sistema de producción ${ }^{3}$.

BAUDRILLARD, J.

\begin{abstract}
RESUMEN:
El diseño - como interfaz genérico entre el hombre y el entorno-, y la publicidad — como particular y dominante mediación entre el hombre y los productos-, pueden contemplarse desde un punto de vista sistémico y funcional o bien circunscribirse a una perspectiva simplemente instrumental. En el primer caso priman las consideraciones macrosocioeconómicas, y en el segundo las microestrategias y microtácticas. El marco elegido, el del lujo, es un lugar donde se revelan de manera muy sensible las macrofunciones de ambos mediadores. Tanto el diseño como la publicidad - en cuanto una de sus formas especializadas - pueden ser vistos como técnicas de creación de lujo. Pero hoy también pueden ser entendidos en sí mismos como un lujo, adscrito a una época con un sistema productivo concreto, para cuya revisión se han percibido llamadas ya urgentes.
\end{abstract}

PALABRAS CLAVE: Diseño; lujo; publicidad; ecología; clases sociales.

TITLE: Topic and distopic luxury: A frame for the design and advertising.

\begin{abstract}
:
The design — as a generic interface between man and environment—, and advertising —as a particular and dominant mediation between man and the products - can be seen from a systemic and functional point of view, or circumscribe to just instrumental perspective. In the first case a macrosocioeconomic approaching prevail, and a micro-strategic and micro-tactical in the second. The chosen framework, that of the luxury, is a place where the macro functions of both mediators are revealed in a very sensitive way. Both the design and advertising - as one of its specialized forms - can be seen as luxury building techniques. But today also they can be understood in

${ }^{1}$ Profesor en la Facultad de Ciencias de la Información de la Universidad Complutense, donde imparte Identidad Visual Corporativa, Introducción al Diseño Gráfico e Historia de la Comunicación Publicitaria. Director del TDGP (Taller de Diseño Gráfico Permanente). Miembro del BEDA (Bureau of European Designers Associations) desde 1981. Socio de AEPD (Asociación Española de Profesionales del Diseño). Socio fundador de di-mad (Asociación Diseñadores Madrid).Correo electrónico: gonsolas@ccinf.ucm.es.

${ }^{2}$ McKendrick, N.; Brewer, J.; Plumb, J. H.: The Birth of a Consumer Society: The Commercialization of Eighteenth-Century England, London, Europa Publications, 1982, cit. por CAMPBELl, C.: The Romantic Ethic and the Spirit of Modern Consumerism, Oxford (UK)-Cambridge (USA), Blackwell, 1987, 21.

${ }^{3}$ BAUDRILLARD, J. (1972): Crítica de la economía política del signo, México, Siglo XXI, 1974, 156.
\end{abstract}


themselves as a luxury, attached to a period with a productive specific system, for whose review, already-urgent, calls have been perceived.

KEY WORDS: Design; luxury; advertising; ecology; social classes.

\section{Introducción}

Hablar del lujo parece de mal gusto. Es un discurso «incorrecto». Del lujo no se habla, simplemente se disfruta, o se observa desde una complicidad tácita. El lujo marca un dentro y un fuera, por lo que genera exclusión y temor a la exclusión, a la no pertenencia al grupo. Este esquema podría ser teatralizado hoy como el discurso del plebeyo postindustrial frente a los seductores reflejos de la sociedad cortesana aún vigentes.

Sin embargo solo desde la posición del extraño se puede hablar del lujo. Porque por un lado el posicionamiento crítico siempre tiene carácter autoexcluyente, des-integrador. Y por otro, la democratización del lujo nos ha convertido a todos en desclasados: a unos porque su clase ya no es sino un modelo codificado para advenedizos, a otros porque lo que se copia del modelo es siempre un simulacro que contradice al modelo mismo. Desde un punto de vista racionalizador se podría hablar de la disolución del lujo.

No obstante parece que el tema ha cobrado cierta actualidad. Por una parte la bonanza económica de los últimos años ha hecho que el acceso a la mercancía se haya transformado en un derecho que unos, acomodados, disfrutan sin sobresalto, otros reclaman como meta de una carrera emprendida, y a muchos moviliza hacia supuestos paraísos de abundancia prometida: es el lujo como horizonte. Por otra parte, la saturación de bienes materiales estereotipados ha forzado a imaginar otras fórmulas de exclusividad: se trata de la distinción como motor. Y por fin, el consumo descontrolado de algunos bienes ha hecho apreciar su terminal escasez, creando nuevas formas de lujo: es el antilujo testimonial.

En esta somera introducción van ya implícitos ciertos componentes constantes de lo que se ha estimado como lujo a través de la historia, aunque las fórmulas han variado. En cierto modo esto parecería llevar a concluir que el lujo es algo permanente e inevitable para la naturaleza humana, compartido incluso, según algunos datos de la etología, con otras especies animales. Sin embargo conviene, ante todo, separar esos componentes para luego ver las relaciones entre ellos, donde podrá apreciarse más una dinámica dialéctico-histórica que determinista, más contradictoria que antinómica ${ }^{4}$.

La propuesta que aquí se intenta ensayar es la siguiente: cada uno de los componentes del lujo cumpliría una función propia, al tiempo que entre las varias funciones se detectaría una jerarquización según la cual algunas operarían como legitimatorias de las otras. El diseño y la publicidad aparecerían actualmente como traductores y legitimadores dominantes de fórmulas tradicionales del lujo.

Estas líneas están concebidas como marco general en el que no se trata precisamente de aclarar la verdadera naturaleza del lujo y sus posibles variantes — que sería un

\footnotetext{
${ }^{4}$ JAMESON, F. (1994): Las semillas del tiempo, Trotta, Madrid, 2000.
} 
simple ejercicio clasificatorio o de agudeza narcisista-, sino de rastrear su estatuto social en la actualidad. De ese modo se propondría descabalgar tanto al diseño como a la publicidad de aquellos discursos tan habituales que prescinden del marco condicionante de su operar, y parecerían autolegitimarse como espacios con total autonomía, incluso «científica».

\section{Crítica de las funciones del lujo}

El lujo es una de las formas de consumo. La literatura específica sobre el lujo es variada y abundante, y por lo general siempre remite a ciertos autores considerados obligados. Pero si se atiende sobre todo a la perspectiva del consumo la literatura relacionable con el lujo es creciente.

La frustrante Memoria sobre el lujo, promovida por Diderot como base para el respectivo artículo en la Enciclopedia, ya recogía la mayoría de las variedades del lujo, así como las posiciones a favor y en contra ${ }^{5}$. Sin ánimo de suscribir la «argucia del enterrador», por la que se declara obsoleta cualquier argumentación no conveniente, es al menos oportuno decir que las nuevas circunstancias han convertido aquellas especulaciones en excesivamente limitadas a sus condiciones históricas. Los cambios generados últimamente en las relaciones del hombre y su entorno parecen exigir un cambio radical en el análisis del lujo.

Para lograr otra perspectiva conviene ante todo describir una cierta estructura permanente en el campo del lujo. En el presente discurso se ha reducido a tres factores determinantes, que no son simplemente taxonómicos, sino que se relacionan entre sí de manera jerárquica y dialéctica ${ }^{6}$.

\subsection{La función antropológico/mítica: la legitimación}

La primera parte de este rótulo parece algo heterogénea, e incluso contradictoria, ya que uniría lo que empíricamente es considerable como propio de la especie con lo que no sería sino una construcción autoexplicativa. Pero tiene su validez operativa y des-

\footnotetext{
${ }^{5}$ JuRADO SÁNCHEZ, J. (2007): «¿Qué era lujo y qué necesario en la Edad Moderna? El debate sobre el consumo suntuario en la literatura europea y española del siglo XVIII», V Encuentro Ibérico de Historia del Pensamiento Económico, Madrid 12-15 diciembre. http://bit.ly/aD7R9M. Web visitada el 18/1/2009.

${ }^{6}$ La división tiene relación, como es lógico, con el campo del consumo. Por ejemplo, Carlos Soldevilla distingue tres perspectivas del consumo, que coincidirían con una posible clasificación de la literatura sobre el lujo. En una perspectiva que llama «apocalíptica», en la que el consumo se construye desde la oferta, se situarían autores como Marx, Veblen, Baudrillard, Jameson, Bauman o De Certeau. En otra, que llama «anfibológica», y en la que el consumo es visto como un cierto encantamiento, sitúa a Simmel, Weber, Benjamin, Bourdieu o Ritzer. Y en una perspectiva «apologética» incluye a Spencer, Douglas-Isherwood, Bataille, Maffessoli, Passeron o Featherstone.

Soldevilla, C. (2001): «Triálogo. Aproximaciones teóricas a la sociología del consumo», Cuadernos de Realidades Sociales, 57-58, Madrid, 13/73.
} 
criptiva. En conjunto se trata de un factor nuclear, que parece enraizar en la naturaleza humana a través de las manifestaciones más primitivas de la culturización de la misma.

Este factor está representado de manera especial por autores como Mauss, Bataille, Elias, o Harris ${ }^{7}$. La denominación de antropológico deriva de que el lujo aparece como algo siempre detectable en la historia de la humanidad, y remite a una funcionalidad no inmediata, sino más bien de tipo simbólico e incluso mítico. La práctica representativa más estudiada es la del potlatch (pronunciado potlok), que se ha convertido en un término tópico que resume esta función. Proviene de la práctica de ciertos indígenas de la costa oeste del subcontinente norteamericano, quienes periódicamente destruían posesiones y riquezas, con una funcionalidad muy compleja, pero que Mauss cristalizó en torno al concepto de don, o de intercambio simbólico. Desde una función primaria de mantenimiento de un orden y un equilibrio en y con la naturaleza se seguía la virtualidad de estructurar la sociedad sin conflictos violentos, mediante lo que en cierto modo podría llamarse violencia simbolizada y sublimada, determinando jerarquías e impidiendo señoríos perpetuados fuera del mérito personal, entre otras virtudes. Práctica ejercida siempre, sin embargo, en un contexto agónico.

Aunque para Mauss se trataba de un «hecho social total», y, por lo tanto, inmanente, no hay impedimento para el desarrollo hacia lo mítico, ya que la destrucción de bienes en un contexto ritual-festivo establecía una relación contradictoria (como todo mito) con la naturaleza. La eliminación y derroche de bienes no deja de tener un componente de hybris, de exceso, de pasarse de la medida, tal vez derivado de una angustia sobre el ser y el porvenir. El concepto de hybris (hiper-, super-: soberbia) lo elaboraron los griegos como concepto negativo y de límite: cualquier pretensión de creerse un dios atraía la envidia y consiguiente amenaza de los dioses (aquellos dioses de los que se ha dicho que eran más mezquinos que los humanos, por lo cual los griegos no tuvieron los dioses que se merecieron), por lo que convenía mantener cierta mesura en pretensiones y posesiones. El potlacht en cierto modo podría ser una meta-hybris, una hybris de segundo grado, contrarréplica por la que el hombre se igualaba a los dioses pero sin ofrecer motivo para su venganza: se destruía para no ser como dioses, pero con ello se mostraba o alardeaba de ser capaz de reconstruir, de recrear. Era un reto al destino y a la naturaleza (cuando la naturaleza aún se pensaba como renovable de forma inagotable). De modo que, además de lo que desde Bataille se ha denominado «lo positivo de la pérdida», pueden detectarse otros aspectos que no son precisamente de ganancia sino

${ }^{7}$ Mauss, M. (1968): «Ensayo sobre los dones», Sociología y Antropología, Madrid, Tecnos, 1991; BATAIlle, G. (1933): La notion de dépense, Paris, Le Éditions de Minuit, 1967, 28; EliAS, N. (1982): La sociedad cortesana. Madrid, Fondo de Cultura Económica, 1993. HARRIS, M. (1975): Vacas, cerdos, guerras y brujas: los enigmas de la cultura, Alianza Editorial, 1991.

El número 318, noviembre 2007, de Revista de Occidente se dedica de forma monográfica al lujo. Allí se puede se puede encontrar la bibliografia de referencia más socorrida. De entre sus artículos conviene señalar el de REMAURY, B.: «El objeto de lujo en la era de la reproductibilidad técnica», 78-94, con un punto de vista muy semejante al expresado por el autor del presente artículo en GONZÁLEZ SOLAS, J. (2008): «La función del diseño en el lujo», III Jornadas de Diseño y comunicación, Madrid, Universidad Complutense, en trámite de publicación. 
de rebelión contra la limitación, de «non serviam»: en la sumisión del contrario, en el duelo de regalos consumidos, en la superación de la angustia a través de la acumulación de autoestima, hay también algo de proyección fálica, en cuanto que poder, soberbia y lujuria (luxuria) indican tensiones de superación tanto como de superhombría.

En dirección opuesta al derroche, aunque en una misma constelación de conceptos, se encuentra el deseo, que actúa también como réplica de la distancia entre el sujeto y el objeto. Pero esa tensión de aspiración a la posesión de un valor - que en el fondo sería reflejo de la finitud, vertida como carencia de lo otro-, en la práctica se concreta en valores no absolutos y abstractos sino mediados socialmente. El lujo se formaliza cuando expresa un valor establecido frente a un tipo de carencia también establecida, y sobrepone un individuo a los demás, creando una diferenciación social que destila poder.

Por otro lado, el goce derivado de la anulación de las contradicciones entre el sujeto y el objeto por medio de la posesión — que resuelve una previa oposición aparentemente sistémica ${ }^{8}$ - tiene que ver con un camino que no es el del desafío y el de la hybris, sino el del juego. En ese sentido tiene analogías con el arte, en la manera en que Jauss o Gadamer lo han considerado ${ }^{9}$. En un sentido más funcional el juego del amor es para Sombart nada menos que el origen de todo un sistema económico como el capitalis$\mathrm{mo}^{10}$, mientras para Simmel ${ }^{11}$ — volviendo a las funciones del don, del derroche y de la excepcionalidad-, la coquetería es un juego social, por debajo de los temas serios y por encima del compromiso individual, algo que vertebra y articula la sociedad, en relación con aquella función fática que Malinowski ${ }^{12}$ calificaba también como un juego: el de hablar por hablar.

La magnificencia, que Condillac establecía como una forma de lujo ${ }^{13}$, pertenece también en cierto modo a este enjambre de conceptos adscritos al nivel ritual, que no dejan por ello de tener sus consiguientes reflejos funcionales en situaciones de clase y clasificatorias.

El complejo campo semántico constituyente del factor antropológico es radicalmente ambiguo, lo que ha facilitado su proyección mítica. Esa ambigüedad permite diversas manifestaciones que se concretan a través de las circunstancias históricas y de la acción humana, que las determina como funcionales o disfuncionales para la especie.

\footnotetext{
${ }^{8}$ Sistémica en el sentido estructuralista, pero no de tipo antinómico («o lo uno o lo otro»), que expresaría la insuperabilidad y la imposibilidad de cambio, sino dialéctico, y por lo tanto manejable.

9 JAUSS, H. R. (1972): Pequeña apología de la experiencia estética, Barcelona, Paidós, 2002. GADAMER, H.-G. (1977): La actualidad de lo bello. El arte como juego, símbolo y fiesta, Barcelona, Paidós, 1991.

${ }^{10}$ SOMBART, W. (1912): Lujo y capitalismo: Revista de Occidente, Madrid, 1928.

${ }^{11}$ Simmel, G. (1917): Cuestiones fundamentales de sociología, Barcelona, Gedisa, 2002, 91-92.

12 MalinOwsKI, B.: «El problema del significado en las lenguas primitivas», en OGDEN, C.K.; RICHARDS, I.A.: (1910-22): El significado del significado, Buenos Aires, Paidós, 1964, 334.

${ }^{13}$ Condillac, E. B. de (1776): Le comerce (sic) et le gouvernement considerés rélativement l'un à l'autre. Amsterdam. En su capítulo XXVII, titulado Du Luxe, 228 ss., escribe: «Depuis qu'on écrit du luxe, les uns en font l'apologie, les autres en font la satyre, $\&$ on ne prouve rien». Sin embargo no deja de emitir su juicio cuando clasifica los lujos como de magnificencia, de comodidad y de frivolidad, 235.
} 
Por ejemplo, el componente de hybris puede adoptar una versión colectiva, como la descrita, pero también una individualista y postnietzscheana, según la cual el reto no se dirige a la naturaleza sino a los semejantes, bajo forma de desprecio a los timoratos, o como elaboración de un yo titánico que se autolegitima en su intransitoriedad: una versión muy propia de nuestra época de construcción de identidades protésicas.

Por la misma razón, los términos empleados (deseo, placer, arte, juego, amor...) son sumamente peligrosos, susceptibles de ser empleados tanto para defender como para denostar el lujo, según la posición ocupada por el enunciador. Son términos favoritos de la publicidad, autolegitimada siempre por su recurso al mito y su elusión de las mediaciones. Si alguien se deja hoy seducir por una interpretación del lujo desde un non serviam en modo sofista (tantas apelaciones publicitarias a la «rebelión»), no puede dejar de percibirse un aire de tragicomedia o de patetismo a cuyo fondo se detecta la satisfacción del publicitario o la carcajada del mercado. Por esta circunstancia, tras las elaboraciones antropológicas que definen funciones aparentemente inocentes, naturales (o aparentemente inocentes por su naturalización), asoma la sospecha de que tales funciones se articulan de manera real dentro de una organización social que establece en concreto ciertas carencias como objetos de deseo. Los sujetos deseantes son la correlación de un sistema de mercancías, tal como expresa Marinas al referirse a la Filosofía del dinero de Simmel:

En el mismo momento en que incorporan los signos del mercado, las equivalencias forman también una red de sujetos deseantes que son el correlato de aquellas mercancías interrelacionadas. La interacción, categoría clave, vuelve a ser redefinida por cuanto se ve coloreada ahora por la relación desigual en el deseo de los bienes y mercancías ${ }^{14}$.

Por esta causa la desigualdad será otro de los marcos interpretativos del lujo, al que la antropología dará paso forzosamente cuando se analice la función distributiva infraestructurante.

La proyección mítica (que eleva los datos a necesidad) aparece también, por ejemplo, cuando se defiende el lujo como motor de la economía (Sombart); o como «goteador» de beneficios colaterales (trickle down effect), cuando se dice que crea empleo o que hace circular el dinero. En realidad el análisis no se está haciendo en el terreno estrictamente económico sino en el mítico: de ningún modo se pone en duda la existencia ni la necesidad del lujo, y así se salva la contradicción entre lujo y economía. Por el contrario, un análisis de contradicciones no deterministas haría ver, por ejemplo, que el lujo del Primer Mundo queda legitimado por esa «beneficencia» racionalizada para mantener las diferencias.

Es posible que también sea en ese mismo espacio mítico —a través de formas simbólicas estandarizadas y naturalizadas - donde se configura cierta socialización de la conducta que puede luego reflejarse en las prácticas. Por ejemplo, aunque pueda parecer muy periférico y exagerado, cabría indagar si la imposición de lo espectacular, o de

\footnotetext{
${ }^{14}$ MARINAS, J. M. (2000): «Simmel y la cultura del consumo», Reis, 89, 183-218.
} 
ciertos elementos estéticos típicos de la «era neobarroca» ${ }^{15}$, podrían ser entendidos como una preeducación para el exceso y el lujo, una transformación del «canon de gusto» en «canon de gasto», lo cual no estaría lejos de las posiciones de Veblen ${ }^{16}$. Pensemos en la espectacularidad de las puestas en escena, donde se construye y valida el «aspecto» de lo moderno para una sociedad de masas a partir de «complementos» (término típico de la moda) accesibles, que, como su nombre indica, son añadidos, sumandos, indicadores de «más», elementos fálico-acumulativos que suelen ir más allá de la simple decoración (decus: lo adecuado, lo conveniente) ${ }^{17}$. De ese modo quizás se construye un modelo conductual en niveles de bajo perfil consumista, con capacidad para autorreplicarse en los espacios del lujo consuntivo de mayor nivel.

El factor antropológico/mítico es reacio a la historia, y tiende a ser considerado como permanente. Ciertas defensas del lujo aparecen a esta luz como de ideología conservadora, la misma que, de manera contradictoria, proclama por otro lado la superación económico-racional de la etapa mítica. Estas contradicciones pueden surgir porque el lujo divide su espacio entre una parte sacral y otra profana. Esta segunda, representada en los otros dos factores que describen el lujo - el estructurante y el distributivo-, busca su legitimación en la primera. La mitificación se convierte en mixtificación de las dos partes, y como todo mito es irreducible a código se dan por «naturales» (irracionales, irreducibles a la razón) las formalizaciones históricas de la parte profana. Es precisamente la solución mítica la que opaca las contradicciones de lo que no se sabe (o no se quiere) resolver en el plano de la realidad. Por eso la última mixtificación operable es la de hacer del lujo una representación anticipada —una utopía — de la felicidad, puesto que en este caso se trata de una felicidad con contrapartida: la exclusión de otros. Sólo en las hipotéticas «nuevas formas» del lujo, como se verá más adelante, se intenta obviar esa contrapartida.

Sin embargo la legitimación operada por el mito nunca logra llevarse a cabo con plenitud y convicción. El verdadero mito (el verdadero deseo) es la supresión de la falta, no el pseudomito de su enmascaramiento. Por eso «En la época en que reina la "felicidad despótica", los individuos ya no se limitan a ser desdichados, ahora se sienten culpables por no sentirse bien» ${ }^{18}$. Buscar la trampa es más una patología que una solución.

La experiencia parece confirmar que el discurso legitimatorio es el más habitual (tanto en diseño como en publicidad) ante las objeciones de carácter racional, y por ello precisamente se adscribe al discurso mítico. El discurso habitual del lujo permanece aún en un territorio no desencantado por «lo moderno», y parece haber pasado intacto a «lo postmoderno».

\footnotetext{
${ }^{15}$ CAlabrese, O. (1987): La era neobarroca, Madrid, Cátedra, 1989.

${ }^{16}$ Veblen, T. (1899): Teoría de la clase ociosa, México, Fondo de Cultura Económica, 1971, $121 \mathrm{ss.}$

${ }^{17}$ En la puesta en escena de multitud de telefilmes televisivos de temática juvenil, el retrato robot de los personajes se aleja lo más posible del grado cero retórico, de la «in-significancia».

${ }^{18}$ LiPOVETSKY, G. (2006): La felicidad paradójica. Ensayo sobre la sociedad de hiperconsumo, Barcelona, Anagrama, 2007, 323.
} 


\subsection{La función socio-estructural: la exclusión}

Si se desciende de la confrontación entre lujo y naturaleza a través del mito y del rito, y se aborda en su relación con la sociedad y su estructuración interna, entonces aparece su contenido fenoménico de tipo diferencial, es decir que marca o produce diferencias. Estas diferencias, al igual que en el factor antropológico, por un lado establecen estratificaciones funcionales y por otro introducen conflictos. En paralelo con las clásicas divisiones sociales y técnicas del trabajo, el lujo produce también divisiones - bien técnicas, bien sociales-, en la apropiación y disfrute de ciertos recursos exclusivos. Sus efectos se manifiestan en paralelo a la división weberiana de clase, estatus y poder, algo que ya Kant expresaba como posesión, fama y poder ${ }^{19}$.

La clase «clásica» (aunque la de Weber, no la de Marx), entendida como cúmulo de posesiones materiales, estructura la sociedad mediante el acceso a distintas posibilidades en la misma. La clase no va necesariamente ligada al prestigio, pero puede ser una de las vías hacia él, aunque no siempre convalidada por él, como se manifiesta en términos como «advenedizos», «nuevos ricos» o «desclasados». La clase resulta ser, en sociedades no estamentales, el sistema de diferenciaciones menos estable, al que se adscriben categorías tales como la movilidad social, el mérito, el esfuerzo personal... Pero aunque pudiera parecer la categoría de estructuración social más democrática nunca ha resuelto la «igualdad de oportunidades», que abarcaría no sólo los puntos de partida individuales sino también los familiares e históricos, que son los que aportan diferencias difícilmente domesticables. El lujo sería una expresión sintomática y expresiva de esta desigualdad, ya que el sentido de clase es equivalente al de distancia social. El lujo incide y señala la distancia de clase estructural, y también marca a quien tiene «clase» y a quien no.

La excepcionalidad o exclusividad del lujo proporciona también un prestigio o estatus, con su correspondiente efecto estructurante de la sociedad. Aunque es el prestigio la categoría más vinculable con el factor antropológico, de hecho es también una categoría elaborada dentro de la sociedad misma, y, por tanto, no de ascendencia mítica o natural, sino histórica y convencional. Sin embargo la legitimación del estatus tiende a hacerse mediante la naturalización de algo que se considera original y dado, no construido artificialmente. Se tiende a la consolidación de tipo estamental, buscando la legitimación en ese supuesto innatismo situado en el terreno de lo antropológicomítico.

En cuanto al poder, por un lado puede ser considerado como consecuencia de alguno de los elementos estructurantes anteriores, o de ambos, pero a su vez puede llegar a generarlos y a vehicular intereses de clase o de estatus. Al igual que la clase alude a distribución económica, el poder remite a objetivos concretos: ambos pertenecen al orden de lo racional o racionalizable. Frente a ellos el estatus queda en esa zona ambigua entre término aspiracional y fuente de legitimación.

\footnotetext{
${ }^{19}$ WeBer, M.: Ensayos de sociología contemporánea, Barcelona, Planeta Agostini, 1985, 145-146.
} 
La relación del lujo con estos conceptos es clara si advertimos en el lujo un factor de distinción y separación, algo cuya distribución, por definición, no es igualitaria en ninguno de los tres sistemas de estructuración social citados.

Veblen, Bourdieu, Simmel o Lipovetsky, son autores frecuentemente citados en este entorno temático del lujo. Todos ellos parten del análisis de diferencias que polarizan situaciones en uno de cuyos extremos se encuentra el lujo. El lujo es un concepto polar, que habla del disfrute de una producción generada en el otro polo (Veblen), de la producción social de las diferencias en la apreciación de los bienes (Bourdieu) ${ }^{20}$, de la adscripción grupal o individual a formas de apariencia exclusivas y excluyentes (Simmel). La pretensión de ruptura de esa polaridad parece nadar siempre en la mixtificación, o recae en una legitimación derivada de la visión mítica que intenta compensar las contradicciones (Lipovetsky).

De nuevo, es la visión mítica la que intenta legitimar estructuraciones que no son sino históricas. Y es precisamente en la coyuntura de las decisiones cotidianas donde actúa el modelo mítico, sustentado permanentemente por métodos de violencia simbólica (la publicidad por ejemplo), de modo que, por ejemplo, «en el enfoque del consumo la categoría de pertenencia - a un status en Veblen, a un estilo en Simmel- es central desde el principio» ${ }^{21}$. Una de las argucias más frecuentes (probablemente cínica) es la de adjudicar al lujo (entendido como término polar del consumo y la apropiación) una función de estructuración social, basada últimamente sobre opciones de psicología individual (permanentes, naturales: míticas), ocultando que sólo significa una desigualdad establecida en otros sistemas coyuntural e históricamente estructurantes, pero no antropológicamente necesarios o determinantes. Hoy es el sistema de mercado el que organiza el sistema del lujo. Por lo tanto, sigue siendo la historia, y no el mito, quien ordena el factor socioestructurante del lujo.

\subsection{La función distributiva: élite y personalización}

Esta función remite sobre todo a la componente económica del lujo, que, aunque muy imbricada con las otras, tiene alguna característica diferenciadora. Si bien el objeto convencional de la economía - la occidental, como economía por antonomasiano es precisamente el lujo, éste podría ser considerado como un horizonte o límite desde el que se ordenarían las diversas categorías de bienes y accesos. Desde este supuesto, sólo aparentemente heterodoxo, podría entenderse que las posiciones frente al lujo se reflejan en la práctica de la administración y distribución de recursos en que consiste la economía. Las opciones a este respecto suponen modelos, unos claramente ideológicos, y otros sólo hasta hoy aparentemente aceptables.

Algunas de estas posiciones, como la de Sombart, se han hecho de obligada referencia en la defensa del lujo, aunque la suya no deje de ser en cierto modo pintoresca,

\footnotetext{
${ }^{20}$ BourdieU, P. (1979): La distinción : criterio y bases sociales del gusto, Madrid, Taurus, 1991.

${ }^{21}$ MARINAS, J. M., op. cit.
} 
tanto por aventajar en misoginia a Marinetti como por contradecir a Weber ${ }^{22}$. Su libro Lujo y capitalismo iba a ser titulado en principio Amor, lujo y capitalismo, y en él sitúa a la mujer, y a las nuevas tendencias surgidas en el paso a la sociedad cortesana, como origen de un resurgimiento económico basado en las industrias del lujo vinculado al amor cortesano. «Así, el lujo, hijo, como hemos visto, legítimo del amor ilegítimo, es el generador del capitalismo». Y su postura no está lejos de la más cínica de Mandeville y su «private vices, public benefits» ${ }^{23}$. Más allá de lo acertado o no de sus estimaciones, lo que evidencian es la ausencia de interrogantes sobre el origen mismo del lujo y de su valor de síntoma de una forma de estructurar la sociedad ${ }^{24}$. En los detalles documentales aportados por Sombart se percibe la restricción del análisis a lo inmediato y fenoménico y no a lo genético y causal-histórico ${ }^{25}$. Pero sobre todo se constata la tendencia a anclar la justificación y legitimación en elementos de tipo psicológico, individualista y autorreferencial, muy al uso hoy en ciertas acepciones de lo postmoderno. Es difícil optar por la teoría freudopsicológica de Sombart (lujo-refinamientoeros) frente a la marxistajungiana de poder fálico, pues en realidad el lujo no se opone a la cobertura de las necesidades sin refinamiento, sino más bien a la escasez y al sometimiento: el lujo tiene contrapartida, como el capitalismo mismo. Y no como antinomia insuperable, natural, sino como contradicción histórica y resoluble. Puede que en un primer momento (mercantilista) el «provecho» capitalista provenga del lujo (productos exóticos, producción costosa y especializada), pero cuando el beneficio procede del gran mercado actual el lujo ya no se sostiene por razones de pura economía, sino sólo como un segmento más del conjunto del mercado (en ascenso, por cierto, en estos úl-

\footnotetext{
${ }^{22}$ MARINETTI, en su primer Manifiesto, tras glorificar la guerra glorifica también el desprecio a la mujer y propone combatir el feminismo: «...hablamos en nombre de la raza, que exige hombres ardientes y mujeres fecundas [...] Toda mujer bella, dejando a las ancianas y a las feas el lujo como única defensa, debe inventar una forma de vestido propia y confeccionarla ella misma, haciendo así de su cuerpo, sencillamente adornado, un originalísimo poema viviente» («Contra el lujo femenino». 11 marzo 1929: Revista de Occidente, 318, 159-163, 2007). Sombart en cambio pone a la mujer en el origen no sólo del lujo sino del origen de capitalismo, frente a la tesis de Weber que lo sitúa en la tendencia contraria, la del ahorro, promovida por el calvinismo: WeBER, M. (1903): La ética protestante y el espiritu del capitalismo, Barcelona, Península, 1994. SOMBART escribe en la misma época que Marinetti, y su ambivalencia le ha hecho ser considerado tanto anti como pro-nazi, e incluso ser vetado como marxista. En ello tuvieron que ver dos de las obras que compondrían su trilogía sobre El Capitalismo moderno, en una de las cuales trata de los judíos y en la otra del lujo.

${ }^{23}$ Mandeville, B. de (1723): The Fable of the Bees: or, Private Vices, Publick Benefits, London, Bible and Crown.

${ }^{24}$ Así se evidencia en citas como «para desquitarse del tedio que les abruma, hacen dos toilettes al día»: MERCIER, L-S.: Tableau de Paris, cap. XC , citado por SOMBART, op. cit., 57 (a este respecto MARINETTI hablaba de «toilettismo»); o en la creación de «academies de femmes aimables»; o en la relación de gastos de lujo de la corte de Francisco I, 1542, donde el apartado «menues plaisirs» supera a todos con un $25 \%$; o cuando dice que en la ciudad «el lujo privado viene a quedar sustituido por el lujo colectivo».

${ }^{25}$ Algo que no falta en otro tratadista como EliAS, N. op. cit.. Aquí se evidencia mejor la función del lujo como adscrito a una forma social e histórica concreta, lo que sugiere que, en caso de que ese hecho social se prolongue o perpetúe en otras formaciones sociohistóricas, habrá que explicar de nuevo su función en ellas.
} 
timos tiempos, a pesar — ¿o quizás incluso en contra? — de la última crisis). Se puede decir que la posición de Sombart es premoderna, en el sentido de ignorancia del límite, y moderna en su pretensión racionalizadora a través del capitalismo.

Más actualizado, pero en la misma posición, parece encontrarse Cutolo ${ }^{26}$, en cuanto a concebir el lujo como motor de la economía, aunque cierta mala conciencia derivada ya de la percepción del límite y de las disfunciones del lujo le hacen ensayar una legitimación ajena a la racionalidad económica: una especie de ética profana e individualista, el «hedonismo virtuoso», que viene a resultar una especie de hedonismo ilustrado. Algo parecido a lo que ya antes había defendido Berkeley desde la moral, que argumentó Hume desde el refinamiento y el placer, y que más recientemente elabora Campbel1 ${ }^{27}$, en un ejercicio que, según éste, complementa a Weber, pero que, por otro lado aporta materiales justificatorios para quienes, como Cutolo, o Ritzer ${ }^{28}$, quieren reivindicar la permanencia del lujo bajo formas benévolas, o incluso a veces bajo nuevas formas conservadoras.

La mayor parte de los autores citados se adscriben más a posturas constatativas que críticas, ya que en general omiten las causas históricas e infraestructurales del modelo que exploran, o pretenden consolidar un modelo que sólo se ha generado en una circunstancia concreta pero que no tiene por sí mismo garantía de funcionalidad social. Su modelo es de tipo expansivo, «colonial», en su tendencia a empujar las fronteras, pasando de las geográficas a las mentales, y del lujo de élite al lujo de masas (en realidad un oxímoron): modelo coincidente con el estándar publicitario. Los procesos de distribución de bienes más allá de las características físicas (marcas, intangibles...), acudiendo a la coartada de lo emocional y de la personalización, significan un cambio de territorio pero no de modelo, que, en la actualidad, parece haber topado ya con alertas sobre ese mismo modelo ${ }^{29}$.

${ }^{26}$ Cutolo, G. (2003): Lujo y diseño, Barcelona, Santa \& Cole, 2005.

${ }^{27}$ CAmpbell, C. (1987): The Romantic Ethic and the Spirit of Modern Consumerism, Oxford (UK)Cambridge (USA), Blackwell. Desde el nivel que parece concederle el pretender complementar-contradecir a Weber, Campbell es uno de los más conspicuos apoyos de las corrientes basadas en el «prerromanticismo calvinista» y en el individualismo burgués como origen del capitalismo de consumo., como se manifiesta, por ejemplo, en Morace, F. (1990): Contratendencias. Una nueva cultura del consumo, Madrid, Experimenta, 1993.

${ }^{28}$ RITZER, G. (1999): El encanto de un mundo desencantado. Revolución en los medios de consumo, Barcelona, Ariel, 2000.

${ }^{29}$ El lujo como motor de la economía reduce toda consideración a la clase, siguiendo con la terminología de Weber, y parece olvidar las categorías y el factor estructurante del prestigio y del poder. Aun así el poder del lujo dentro de la economía resulta ser tan grande e ineludible que, dentro de la literatura del diseño, incluso los escritores más críticos, como Maldonado y Bonsiepe, cuentan con él. MALDONADO, T. (1997): El diseño industrial reconsiderado, Barcelona, Gustavo Gili, 1993, 61; BonSIEPE, G. (1975): Diseño industrial. Artefacto y proyecto, Madrid, Alberto Corazón, ed., 85. Ambos parecen no poder afrontarlo mediante una prefiguración o representación que lo elimine, y optan por un reformismo que transige con un mal al parecer ineludible-mítico (crear desde el Tercer Mundo productos de lujo para el Primer Mundo), opción cuya única defensa parecería ser simplemente táctica: la creencia en la ausencia de «condiciones objetivas», una cuestión de oportunidad. Aunque se percibe también la nostalgia por la ocasión pedida. Sin embargo esas condiciones se han ido objetivando como «contradicciones» del sistema. 


\section{Algunos datos para el marco}

\subsection{La exclusión lingüística}

Como ya se ha señalado, cuando aún existía capacidad física de expansión de fronteras, como ocurría en la época colonialista, la distancia de los hechos o la lentitud de las comunicaciones procuraba cierta opacidad que facilitaba el mirar hacia otro lado y fabricar una moral filistea. Hoy el mundo se ha estrechado, convertido en aldea global, y casi no quedan fronteras físicas que empujar (¿salvo quizás en la Amazonía, o en los polos a punto de «liquidación»?). Pero se han encontrado nuevas fronteras, no de tipo físico sino psicológico, sobre las que constantemente ejercen presión la publicidad o el marketing, además del diseño, convertido para ello en gran parte al mismo lenguaje de la publicidad. Porque una de las formas de exclusión, que legitima con gran eficacia la exclusión cuantitativa o física, es el uso de un lenguaje adecuado al lujo. Es sobre todo en el lenguaje, y por tanto en el nivel simbólico, donde se opera la legitimación de las funciones subalternas.

Cada club de gusto impone para hablar del lujo un lenguaje que excluye a los demás. El lujo en sí mismo puede ser un lenguaje de poder: autoriza y desautoriza, incluso marca como perteneciente al grupo, de modo que hace participar en la legitimación del grupo mismo, en sus ritos y sus prácticas (incluso innobles) estructurantes, negando la palabra a la crítica externa al círculo mismo del lujo. Quien critica no es del grupo, «no entiende». Por eso se advertía al comienzo que hablar del lujo puede ser un índice de lenguaje incorrecto, de mal gusto, incluso un rasgo plebeyo, provinciano con respecto al «centro» constituido, o un índice de resentimiento. Esta connivencia grupal tiene que ver probablemente con lo que C. Wright Mills estudió como sistemas de poder de las élites ${ }^{30}$. El lenguaje actúa como violencia simbólica con la que excluir todo tratamiento crítico de algo que va de soi, y que parece remitir al nivel antropológico, naturalizado de manera determinista, y convertido, por tanto, en ideología legitimatoria, vía «cuna» o vía éxito, de las exclusiones físicas y materiales. No debería extrañar que el análisis del mapa distributivo mostrase que los distribuidores del excedente material (el lujo como exceso), fuesen también los mismos distribuidores del sentido del lenguaje. Es decir, que la carga significativa del lenguaje fuese del mismo tipo que la carga material, que el lenguaje actuase como plano de la expresión de los hechos materiales, verdadero plano del contenido, y ambos con ejes estructurales semejantes (integraciónexclusión // riqueza-pobreza) consecuencia de un mismo origen sociohistórico. El lujo, y la manera de hablar de él y de usarlo, es un capital in-corporado, propio de una clase cuya reproducción depende de la transmisión de ese capital: que esa clase tenga conciencia de sí como tal, o que esté dispersa, no viene al caso. A esa legitimación Weber la llama «teodicea de su propio privilegio». De hecho puede decirse que existe una articulación entre los componentes materiales y clasificadores del lujo a través del len-

\footnotetext{
${ }^{30}$ Mills, Ch. W. (1963): Power, Politics and People, New York, Oxford University Press.
} 
guaje como sedimento y expresión de un «capital cultural». En el lujo se exhibe un saber: qué vajilla hay que tener, qué hotel frecuentar, «de quién» se ha de vestir... Un saber que se manifiesta en los hechos y en el lenguaje, y que, a través de éste, se apropia incluso de las personas en cuanto vehículos de la prestación de un servicio exclusivo («mi modisto»), en cuanto integradas en un «ocio vicario» ${ }^{31}$. Es ese sentido de apropiación y distribución del lenguaje el que permite decir a Simmel que el lujo, como la prostitución, cuanto más alto es menos justificación moral exige, puesto que sintoniza con la moral construida por sus practicantes ${ }^{32}$.

La defensa del lujo estaría, por tanto, bajo la sospecha de que sólo la existencia de ciertos intereses generaría un lenguaje legitimatorio de los mismos. De hecho, cuando la exclusión material ya no es efectiva se inventa (y se pone de moda) un nuevo lenguaje del lujo. Este sucesivo desplazamiento del campo semántico, o las sucesivas resemantizaciones del mismo hecho, es quizás lo que ha dado pie para enunciar ese supuesto «eterno retorno del lujo» ${ }^{33}$.

La moda, por ejemplo (ejemplo sintomáticamente ineludible cuando se habla del lujo), es una traslación continua del campo de significación superficial, mediante una combinatoria de elementos materiales exclusivos, excluyentes, escasos... que plantean modos de acceso de carácter distributivo; apoyada en una indesmontable tendencia humana a la distinción según modelos preestablecidos, de movilidad ascendente: estructurantes; y legitimada desde campos semánticos adyacentes como el arte, la genialidad, la extraterrenalidad...: legitimidad antropológico-ideológica. Actúa como una gran metá-fora, algo cuyos elementos de coincidencia-legitimidad están puestos más allá, precisamente allí donde no se pueda percibir el más acá: es decir, el origen del sistema material y de lenguaje. Así al menos lo vio Barthes ${ }^{34}$ cuando habló del sistema de la moda: el sistema consiste en que todos sus elementos pertenecen a un solo significado final, una idea del mundo. Más allá de Barthes queda el averiguar de dónde surge tal idea del mundo, algo que, incluso después de Marx, está aún sobre la mesa. Pero una insatisfactoria respuesta no autoriza a sustituir la misma por una ficción, un simulacro, por muy espectacular que sea. La condición humana sigue sin ser descifrada, pero sólo cierto postmodernismo relativista (¿«lógica cultural del capitalismo tardío» ${ }^{35}$ ?), se permite ofrecer sucedáneos de respuesta.

El «lujo democrático», transformado incluso en estilo de vida, se legitima por el sistema mediático (diseño, publicidad...), que es ya el sistema del lenguaje ${ }^{36}$. Se legitima

${ }^{31}$ VEBLEN; Th., op. cit..

32 Simmel, G. (1900): La filosofía del dinero, Instituto de Estudios Políticos, Madrid, 1976, 477-78. A veces la exclusión lingüística se solidifica en exclusión física y geográfica, como sería el caso de la gentrification o de las gate communities.

${ }^{33}$ LoZANO, J. (2007): «El eterno retorno del lujo», Revista de Occidente, 318, noviembre, 5-8.

34 BARTHES, R. (1967): El sistema de la moda, Barcelona, Gustavo Gili, 1978.

35 JAMESON, F. (1984): El posmodernismo o la lógica cultural del capitalismo tardío, Barcelona, Paidós Ibérica, 1991.

${ }^{36}$ Cutolo parece aludir a este aspecto, sin desarrollarlo: op. cit., 43. Sin embargo cabe una interpretación de las relaciones entre diseño gráfico (sistema de la comunicación) y diseño industrial (sistema de 
como normalidad en el foro público, puesto que son los medios de comunicación de masas quienes establecen la normalidad: pasarelas, ferias, exposiciones, premios... que hacen perder la vergüenza y generarla en el opositor, tachado de profano. En la moda, por ejemplo, las pasarelas de alta costura son la fabricación simbólico-mediática del deseo para el prêt-à-porter, que es el lujo democrático acogido al efecto legitimador y semantizador del objeto-marca «auténtico».

La apropiación del lenguaje del lujo proyecta un complejo de inferioridad que nadie quiere padecer, por lo cual induce - por temor a la exclusión y sin que medie la crítica-, a la obtención del estatus de lujo propuesto. Una verdadera violencia simbólica a través del lenguaje.

\subsection{Las teorías de las necesidades}

Las teorías economicistas del lujo corresponden a circunstancias ya pasadas, en supuestos de recursos inagotables y de fronteras físicas expandibles de manera colonial. Las nuevas circunstancias obligan a cambiar estos presupuestos. Incluso convierten casi en prescindibles todas las consideraciones de tipo ético o político, puesto que ya, hoy, la cuestión de los límites se ha hecho presente de manera física e ineludible, hasta el punto de que podría estar en crisis la habitual paradoja del lujo como superfluo y estructural a la vez ${ }^{37}$. Este proceso de reinterpretación, y quizás de ruptura histórica, pasa por una vuelta al análisis de las necesidades y de su construcción social, más que por una insistencia en el análisis de los productos de lujo considerados como abstracciones autónomas. Y este mismo proceso se refleja también en el lenguaje, en el que ciertos términos antes tabú, como el de «necesidad», comienzan a ser utilizados sin el anterior rubor inducido por presiones pseudopostmodernas.

La relación del lujo con las necesidades es recurrente. Sobre todo si nos referimos al Primer Mundo no puede resultar indiferente una afirmación como: «Vivimos en el lujo y simulamos las necesidades $»^{38}$. Y, aun si a una tal llamada de atención siguiese una reacción positiva, no cabe duda de la dificultad de construir un proyecto válido ${ }^{39}$.

El tratamiento de las necesidades con relación al tema del lujo tiene una vertiente socioeconómica, consistente en la administración y reparto de bienes escasos, y otra sociopolítica, que remite a quién define las necesidades, su cuantificación, el sistema distributorio y su adecuación a los derechos previamente definidos.

objetos), según la cual el primero opera como legitimación simbólico-mediática del segundo: GonZÁLEZ SOLAS, J., «La función del diseño en el lujo», op. cit..

${ }^{37}$ Rentabilidad e incertidumbre, peligros paradójicamente no considerados, representación retórica de la realidad (medios de comunicación) que oculta la realidad..., son temas tratados por BECK, U. (1992): La sociedad del riesgo: hacia una nueva modernidad, Barcelona, Paidós, 1998.

${ }^{38}$ SLOTERDIJK, P. : La Vanguardia, Suplemento Cultura 3/5/2007.

39 «Lo que sí veo es un problema de reconstruir los sistemas de necesidades»: ALONSO, L. E. (2004): «El trabajo sin fin», Página Abierta, 151. http://www.pensamientocritico.org/luienralo0904.htm. Web visitada el 2/10/ 2010. 
La senda del deseo, con su carga freudiana y rousseauniana, no ha sido la más oportuna para afrontar el problema de la necesidad y, consiguientemente, de ciertas «necesidades» como el lujo. Lo pulsional parece remitir a cierta naturalización y a un pesimismo esencialista frente a lo racional ${ }^{40}$. A pesar de la fuerza pulsional aún parece quedar lugar para una antropología crítica próxima a la pragmática kantiana, donde puedan distinguirse valores y funciones. El deseo es ilimitado y personal, mientras las necesidades, aunque también personales, son definibles, y las carencias, referidas a los medios, son plenamente cuantificables. Debido a estas distinciones hay quienes, como Len Doyal y Ian Gough ${ }^{41}$, hablan de «necesidades básicas» (salud y autonomía), susceptibles de derechos, y de «necesidades intermedias», plenamente cuantificables mediante indicadores de satisfacción. De un modo parecido Ágnes Heller ${ }^{42}$ emplea los términos de «necesidades radicales», o cualitativas, y «necesidades cuantitativas», además de distinguirlas de los intereses y de las preferencias. Ya que las necesidades radicales o sociopolíticas (libertad, igualdad) no remiten a la escasez, en tanto que las económicas sí, la gestión de la distribución tiene una importancia inmediata. Sin embargo tanto el hiperliberalismo, con su idealización del mercado, como el socialismo autocrático, con su dictadura de las necesidades, no han llegado a estabilizar un procedimiento que no haya generado desigualdades, bien en los aspectos cuantitativos, en el primero, bien en los cualitativos, en el segundo.

Desde una de las perspectivas posibles las políticas distributivas tienen como límite los derechos del otro (el kantiano «que un hombre no sea medio para la posesión, dominio o ambición de otro hombre»), algo que sólo puede admitirse como imperativo categórico. Sin embargo en la realidad es fácil constatar que, de hecho, no todos pueden imponerse a otros, sino sólo los que «pueden». Cualquier análisis de este desequilibrio en las posibilidades nos lleva a consideraciones históricas acerca del origen de las desigualdades de partida, a la atención a la precodificación de necesidades, a los mecanismos de creación de una sociedad insatisfecha, a la educación en las necesidades y satisfacciones, etc. Elementos del marco situacional a los que muchas prácticas del diseño (gráfico e industrial) y de la publicidad, suelen ser ciegos, debido a su fatal y necesaria integración en un sistema preestablecido, aunque este sistema sea sólo una opción, mantenida por lo general como exenta de toda discusión o abordaje científico ${ }^{43}$.

${ }^{40}$ MARINA, J. A. (2007): Las arquitecturas del deseo, Barcelona, Anagrama.

Siempre que en este texto se alude al esencialismo (o su correlativo el naturalismo) se entiende no como opuesto a la volatilidad conceptual, a la pura contingencia transformada en cierto modo en un esencialismo de signo opuesto (antinómico, pero no dialéctico), sino más bien como contrario a la concreción y pragmática históricas. En este sentido la historia se entiende tanto en el sentido local, de circunstancias de producción social concretas, como en su devenir genético o causal inmanente.

${ }^{41}$ DoyAl, L.; Gough, I. (1991): Teoría de las necesidades humanas. Barcelona, Icaria, 1994.

${ }^{42}$ Heller, Á. (1993): Una revisión de la teoría de las necesidades, Barcelona, Paidós Ibérica, 1996.

${ }^{43}$ En relación con la temática distributiva y sus posibles instrumentaciones democráticas conviene recordar las aportaciones de Habermas, en gran parte de su obra, pero en concreto en los debates con Rawls, donde destacan la herencia kantiana y el optimismo rousseauniano: HABERMAS, J. (1996): Debate sobre el 


\subsection{Los nuevos límites: de la metafísica a la ecología}

Lo anterior remite fundamentalmente a una economía clásica y a un capitalismo industrial. Pero el capitalismo postindustrial, el postcapitalismo, el capitalismo tardío, o el postfordista, según se le quiera denominar, estructura la sociedad de una nueva manera hegemónica, relegando a segundo plano componentes de prestigio o poder no radicados en la economía de mercado. A ello se añade la aparición de un capitalismo de consumo que, habiendo alcanzado las últimas fronteras físicas de tipo colonialista, se dirige hacia las fronteras del individuo, y, sobrepasada la etapa de la racionalidad, que supondría un sujeto ilustrado ${ }^{44}$, accede a la etapa de la emoción, en la que se convocan las capacidades irracionales de ese sujeto. Además, la descripción clásica coincidiría con la de un sistema de circulación ideológico-material cerrado y autónomo, como el del Primer Mundo - denominación en sí misma tendenciosa y falaz, aunque habitual—, pero lo que hoy se considera Primer Mundo es ya una ficción en forma de proyecto de extensión global. En estas circunstancias cualquier desigualdad de acceso se transforma automáticamente en dominación y explotación de una parte por otra. Cualquier exceso en las necesidades cuantitativas o intermedias, generado en alguna parte del estrecho mapa del mundo actual, produce exclusiones en otra parte.

Ya se ha señalado que en uno de sus aspectos el lujo es desmedida, derivación a la locura (el dormitorio de Sadam, los zapatos de Imelda, el desvarío del boom edificatorio bajo la coartada de necesidades populares), pero incluso las necesidades cuantitativas o intermedias descritas, una vez satisfechas, se reinventan o se imitan al alza bajo la presión publicitaria, motor del consumo imparable, ley para la sociedad consuntiva. El modelo comienza por la necesidad, sigue por el exceso, y va hacia el agotamiento o hacia el límite, y en su recorrido determina desigualdades en algún lugar, conocido o desconocido.

Las voces de aviso provienen de distintos puntos. Ágnes Heller ${ }^{45}$ afirma que la concepción del proyecto moderno de un progreso universal e ilimitado no puede sostenerse. Luis Enrique Alonso habla de un mercado potencialmente destructivo que institucionaliza consumos como amplios dispositivos de exclusión ${ }^{46}$. Serge Latouche ve incluso el desarrollo como un concepto perverso cuyos efectos pretendidamente generalizables son una impostura, cuando tras más de cincuenta años de aplicación de tal

liberalismo politico / Jürgen Habermas, John Rawls, Barcelona, Paidós: I.C.E. de la Universidad Autónoma, 1998.

${ }^{44}$ «El desperdicio de trabajo humano y de material ha llegado a ser irracionalmente central a la realización del mecanismo capitalista»: MiLLS, Ch. W. (1963): «The man in the middle: The Designer». op. cit., 380-381. Publicado anteriormente en Industrial Design, nov.1958, 72-76. Aún así todo fenómeno tiene sus contradicciones: el otro polo del lujo, el sector empobrecido ¿puede ser también objeto de racionalización bajo el mismo modelo capitalista, pero albergar posibilidades emancipatorias? Ver: PRAHADAD, C. K. (2004): La fortuna en la base de la pirámide: cómo crear una vida digna y aumentar las opciones mediante el mercado, Barcelona, Granica, 2005.

${ }^{45}$ HeLLER, Á: op. cit..

${ }^{46}$ Alonso Benito, L. E. (2005): La era del consumo, Madrid, Siglo XXI. 
concepto el $20 \%$ más rico se ha distanciado mucho más del 20\% más pobre. Su teoría del decrecimiento va más allá del crecimiento cero y de la sostenibilidad ${ }^{47}$. Como desengañado de la larga prepotencia de la ciencia económica se expresa Alfredo Pastor cuando afirma que la desigualdad no sólo es injusta sino insostenible ${ }^{48}$.

Hemos pasado de una etapa en que el horizonte límite era el fantasma de la escasez por falta de dominio tecnológico de la naturaleza, a otra etapa en la que tememos ya la destrucción de la naturaleza por una tecnología ideologizada.

Dejando atrás los presupuestos abstractos y esencialistas -cuando no diletantes y frívolos $-{ }^{49}$, el choque con la pura y mostrenca realidad física, ha transformado la lenta metafísica en ecología urgente.

\subsection{Nuevos lujos}

Hay que constatar, ante todo, que es curioso que ninguno de los proponentes de nuevos lujos se atreva con iniciativas para su radical eliminación. Sin embargo, si uno de los referentes convencionales del lujo podrían ser las 68 empresas afiliadas al Comité Colbert (la patronal del lujo francés), al menos ahora el referente comienza a ser otro: ya no se trata de crisis de la forma de lujo, dejando incólume el hecho, sino de una revisión de sus condiciones de ser, de perdurabilidad. Aunque a veces las propuestas se reduzcan a una resemantización del término.

${ }^{47}$ Un $20 \%$ de los habitantes del planeta consume un $86 \%$ de los recursos del planeta. Si todos sus habitantes quisieran vivir como los españoles, por ejemplo, harían falta, según Latouche, dos planetas y medio. LAtouche, S. (2004): Sobrevivir al desarrollo. De la descolonización del imaginario económico a la construcción de una sociedad alternativa, Barcelona, Icaria, 2007.

El volumen del lujo cifrado por COLE y MiLEs (1984): World Appart, 135-139 (citados por DoYALDouGH, op.cit., 293) excede al de los productos básicos:

Bienes básicos $\$ 3.4$ billones

Artículos de lujo $\$ 5.3$

Bienes de capital $\$ 2.1$

Producción total: $\quad \$ 10.8$

Estas cifras quizás puedan indicar en qué grado las necesidades del 'centro' son parásitas de la 'periferia', y, por tanto, el grado de insoportabilidad a corto plazo, no sólo moral, sino física.

Desde el campo específico del diseño, Papanek, ya en 1971, llamaba a la responsabilidad. PAPANEK, V. (1971): Design for the Real World: Human Ecology and Social Change, Londres, Thames and Hudson, 1985. También el Club de Roma en 1972: MEADOws, D. H. et al. (1972): Los límites del crecimiento, México, Fondo de Cultura Económica, 1982.

48 PASTOR, A. (2007): La ciencia humilde. Economía para ciudadanos, Barcelona, Crítica. El mismo autor también afirma: «Quizá tengan razón los economistas franceses que hablan de decrecimiento. No es un drama tener una economía estacionaria». PASTOR, A. (2008):«Una ciencia contra la desigualdad», El País, Babelia, 23 de febrero, 16.

${ }^{49}$ Es sorprendente que SOMBART no filtre esta apreciación, típica de una estética que se autocalifica por ello, cuando habla desde una perspectiva de teoría económica: «Las demoiselles de París y las misses de Londres habían puesto en pie esta inmensa masa de esclavos para satisfacer sus caprichos. Esta idea no deja de tener cierto encanto» (op. cit., 228). 
Ya para Bataille el verdadero lujo y el potlatch de nuestro tiempo estaba en el miserable, en el que rechaza la riqueza, en el infinito esplendor de la ruina ${ }^{50}$. A partir de las nuevas circunstancias, el lujo ha derivado por un lado hacia una forma de consumo amnésico, disipativo e histérico, cuajado de cierto nihilismo postmoderno, y que a veces pretende conectarse con la propuesta de Bataille y hasta adquirir cínicamente el tinte de una nueva épica. Pero por otro lado ha sido pensado también como otra forma de expresión de la distinción (o de la identidad, pero no precisamente de la desigualdad). Así, Hans Magnus Enzensberger habla de los nuevos lujos del tiempo, de la atención, del espacio, del silencio, del ambiente, de la seguridad, casi todos de tipo opositivo a la corriente principal (mainstream!), en la senda de la apropiación precisamente del concepto de escasez, del que siempre ha pendido el lujo para acceder a la distinción: «El lujo del futuro se despide de lo superfluo y tiende a lo necesario» ${ }^{51}$. Pero esta propuesta, que prescinde de la raíz y del origen de los hechos, corre el riesgo de calcarse fatalmente sobre el modelo anterior, según los parámetros de la moda y la alternancia como sistema simbólico general. Porque una vez hechos escasos el tiempo, el espacio o la seguridad, por la voracidad del modelo de desarrollo, acceder a ellos se convierte en lujo. Domenico di Masi insiste también en la misma línea y reproduce los términos de Enzensberger, aunque añade el valor de la belleza ${ }^{52}$. Romanelli ${ }^{53}$, en cambio, habla del vacío como riqueza de espacio, el lleno (con su peligro de «neorriquismo»), la materia (no en sí misma sino en su forma de uso), el detalle (no exhibicionista sino hecho de pericia y respeto), el recorrido (que hace surgir la narración), y el recuerdo, «el lujo más grande que al hombre le está permitido». Pueden apreciarse aquí fórmulas que no calcan el reverso del modelo de lujo actual, y que por lo tanto no generan formas de exclusión sino de apreciación, procedentes más de una capacidad adquirida a través del acceso a la cultura que a los bienes materiales escasos. Sin embargo es preciso señalar que estas propuestas no siempre son universalizables, ya que son pensadas desde el Primer Mundo occidental o desde una economía capitalista, en la que no entran otras economías, como la del honor, o la del placer autónomo, no consideradas como rentables, pero que apuntarían a una expresión del lujo como soberanía. Es precisamente en ese Primer Mundo donde se produce la paradoja del lujo: éste consiste en la apreciación de los bienes escasos cuya escasez ha sido generada precisamente por los consuntores de esos bienes; desde la naturaleza maltratada (y "publiturística") se ha inducido otra naturaleza residual, escasa, y, por tanto, reapreciada. Los espacios naturales intactos, por ejemplo, son residuos de los espacios consumidos ${ }^{54}$.

\footnotetext{
${ }^{50}$ BAtAille, G. (1949): La parte maldita, Barcelona, Icaria, 1987, 112-113.

${ }^{51}$ EnZENSBERgER, H. M. (1998): Zigzag, Barcelona, Anagrama, 1999. Citado por Manuel Harazem : «El "Homo Luxuriosus"», ARTyCO, 12, Primavera 2001.

${ }^{52}$ CuTOLO, op. cit., 48 y50.

${ }^{53}$ RomanelLi, M. (2006): «Lujo y arquitectura. Vademecum en seis puntos», en Morozzi, C. (ed.): Axolute. Style tech, catálogo de bTicino.

${ }^{54}$ Hay que tener en cuenta que el lujo es un fenómeno local, así como el diseño: la exportación del modelo mercantil a todo el mundo va acompañada de un modelo de diseño que se pretende exportar. Incluso Bonsiepe pretende ser local, pero imita forzosamente a la metrópoli en sus propuestas para el Tercer Mun-
} 
Por eso los nuevos lujos deberían postular un cambio de modelo, no un cambio de objetos de lujo. Y por eso también, como base de cualquier operación de hipotética reconducción del lujo, quizás haya que atender la sugerencia de Latouche de redefinir la idea de riqueza: entenderla como satisfacción moral, intelectual, estética, como empleo creativo del ocio. Por ejemplo, la elección de empleo, o el año sabático a los treinta años, son formas de lujo no competitivo o consuntivo, más allá del «espejo de la producción ${ }^{55}$, y utopías ya realizadas por muchos jóvenes, outsiders de la sociedad de consumo del Primer Mundo. A veces también otras posiciones, en forma de fundamentalismo ecologista, por ejemplo, no hacen sino reproducir modelos esencialistas de signo contrario: no es fácil encontrar el camino de la imaginación que lleve a nuevas formas de satisfacción libidinal no consuntivas ni competitivas.

El sofisma más a mano del inmovilismo ante las propuestas antilujo suele ser la acusación de antiprogreso y de regreso al pasado. Cuando Sombart alude a que «los jaboneros vivían tranquilos y dichosos hasta que un buen día se inventó el jabón de lujo ${ }^{56}$, puede interpretarse también no como un retrato del inmovilismo sino de la felicidad, algo que al menos es comprometido atribuir al lujo. Aunque cierta felicidad también sea atribuible a algunos componentes del lujo. El lujo es también refinamiento, distinción, clase sin clases, excepción, poesía, autogratificación, autohomenaje, evidencia de lo otro, lo festivo, lo no ordinario ni «normal» y, por fuera de la norma, quizás transgresor... La discriminación ética pasaría por saber si el lujo propuesto acrecienta la consciencia o la inconsciencia: la inconsciencia voluntaria ante las disfuncionalidades del lujo siempre pretende dominar el lenguaje para blindarse y hacerse impenetrable, exclusiva y excluyente. O si se trata de lujo privado o público: el privado no puede ser ritual, o no sería estrictamente privado. La divisoria quizás estaría entre el lujo que consume y excluye y el que es inagotable, el cuantitativo y el cualitativo. Tal vez se podría distinguir entre exolujo (riqueza-material-excluyente), autolujo (satisfactoriopersonal-cultural), y heterolujo (inducido por el mercado) ${ }^{57}$.

Quizás los nuevos lujos pudieran surgir a partir de lo que predican algunos coolhunters: dejar de lado el factor exhibicionista y orientarse hacia el placer individual, donde

do: BonsiePE, G. (1993): Del objeto a la interfase. Mutaciones del diseño, Buenos Aires, Ediciones infinito, 1999. Aunque más tarde corrige: «Y de nuevo me pregunto para qué emular modelos de otro lado. Soy escéptico frente a esos trasplantes»: BONSIEPE, G. (2005) «Una charla de diseño», M2. http://www.pagina12.com.ar/diario/suplementos/m2/10-814-2005-11-12.html. Web visitada el 2/10/2010.

${ }^{55}$ BAUDRILLARD, J. (1973): El espejo de la producción, o la ilusión crítica del materialismo histórico, Barcelona, Gedisa, 1980.

${ }^{56}$ SOMBART, op. cit., 265.

${ }^{57}$ En el terreno de la economía de las necesidades y de la distribución de bienes se ha ido evolucionando desde la medida del bienestar por el PIB (producto interior bruto) hacia el IDH (índice de desarrollo humano), en cuya elaboración colaboró el premio Nobel de economía Amartya Kumar Sen), el ISV (índice de satisfacción de vida), o el IDI (índice de desarrollo integral), de Bunge, que incluye el desarrollo democrático. Y así, países con una clasificación por PIB se encuentran en otra por IVS. Es llamativo que un grupo de países difícilmente equiparables en PIB a Francia, por ejemplo, tengan un ISV semejante a ese país, como ocurre con El Salvador, Hong Kong, Indonesia, Kirguistán, Eslovenia, Taiwán, o Timor Este: ver Dobado GonzÁlez, R. (2008): «Sarkozy y otra medición del PIB», Tribuna Complutense, 19 febrero. 
el lujo ya no crearía principalmente disfrute sino un tipo de sujeto, una identidad social. El problema está en qué enunciador se apropia de este lenguaje, en principio ambiguo: si el mercado o el individuo en una democracia mundial real. En este caso sí se podría hablar de «elitismo democrático» ${ }^{58}$.

\section{Referencias bibliográficas}

Alonso BenITO, L. E. (2005): La era del consumo, Madrid, Siglo XXI.

- (2004): «El trabajo sin fin», Página Abierta, http://www.pensamientocritico.org/luienralo0904.htm. Web visitada el 2/10/2010.

BARTHES, R. (1967): El sistema de la moda, Barcelona, Gustavo Gili, 1978.

BatAille, G. (1933): La notion de dépense, Paris, Le Éditions de Minuit, 1967.

- (1949): La parte maldita, Barcelona, Icaria, 1987.

BAUDRILLARD, J. (1972): Crítica de la economía política del signo, México, Siglo XXI, 1974.

- (1973): El espejo de la producción, o la ilusión crítica del materialismo histórico, Barcelona, Gedisa, 1980.

BECK, U. (1992): La sociedad del riesgo: hacia una nueva modernidad, Barcelona, Paidós, 1998.

BONSIEPE, G. (2005): «Una charla de diseño», en $M 2$. http://www.pagina12.com.ar/diario/suplementos/m2/10-814-2005-11-12.html. Web visitada el $2 / 10 / 2010$.

— (1975): Diseño industrial. Artefacto y proyecto, Madrid, Alberto Corazón, ed.

- (1993): Del objeto a la interfase. Mutaciones del diseño, Buenos Aires, Ediciones Infinito, 1999.

BOURDIEU, P. (1979): La distinción: criterio y bases sociales del gusto, Madrid, Taurus, 1991.

CALABRESE, O. (1987): La era neobarroca, Madrid, Cátedra, 1989.

CAMPBell, C. (1987): The Romantic Ethic and the Spirit of Modern Consumerism, Oxford (UK)-Cambridge (USA), Blackwell.

CONDILLAC, E. B. de (1776): Le commerce et le gouvernement considerés rélativement l'un à l'autre.

Cutolo, G. (2003): Lujo y diseño, Barcelona, Santa \& Cole, 2005.

DOBADO GonzÁlez, R. (2008): «Sarkozy y otra medición del PIB», en Tribuna Complutense, 19 febrero.

DoYAL, L.; GoUgh, I. (1991): Teoría de las necesidades humanas, Barcelona, Icaria, 1994.

ELIAS, N (1982): La sociedad cortesana, Madrid. Fondo de Cultura Económica, 1993.

ENZENSBERGER, H. M. (1998): Zigzag, Barcelona, Anagrama, 1999.

GADAMER, H.-G. (1977): La actualidad de lo bello. El arte como juego, símbolo y fiesta, Barcelona, Paidós, 1991.

GoNZÁLEZ SolAS, J. (2008): «La función del diseño en el lujo», III Jornadas de Diseño y Comunicación, Madrid, Universidad Complutense, en vías de publicación.

HABERMAS, J. (1996): Debate sobre el liberalismo político / Jürgen Habermas, John Rawls, Barcelona, Paidós: I.C.E. de la Universidad Autónoma, 1998.

\footnotetext{
${ }^{58}$ «El elitismo democrático es el antimodelo del mundo de crecimiento infinito»: HeLLER, op. cit., 121.
} 
HARRIS M. (1975): Vacas, cerdos, guerras y brujas: los enigmas de la cultura, Alianza Editorial, 1991.

Heller, Á. (1993): Una revisión de la teoría de las necesidades, Barcelona, Paidós Ibérica, 1996.

JAMESON, F. (1994): Las semillas del tiempo, Madrid, Trotta, 2000.

- (1984): El posmodernismo o la lógica cultural del capitalismo tardio, Barcelona, Paidós Ibérica, 1991.

JAUSS, H. R. (1972). : Pequeña apología de la experiencia estética, Barcelona, Paidós, 2002.

JURADO SÁNCHEZ, J. (2007): «¿Qué era lujo y qué necesario en la Edad Moderna? El debate sobre el consumo suntuario en la literatura europea y española del siglo XVIII», V Encuentro Ibérico de Historia del Pensamiento Económico, 12-15 diciembre. http://bit.ly/aD7R9M. Web visitada el 18/1/2009.

LAtouche, S. (2004): Sobrevivir al desarrollo. De la descolonización del imaginario económico a la construcción de una sociedad alternativa, Barcelona, Icaria, 2007.

LIPOVETSKY, G. (2007): La felicidad paradójica. Ensayo sobre la sociedad de hiperconsumo, Barcelona, Anagrama.

LOZANO, J. (2007): «El eterno retorno del lujo», Revista de Occidente, 319, 5-8.

MALDONADO, T. (1997): El diseño industrial reconsiderado, Barcelona, Gustavo Gili, 1993.

MALINOWSKI, B.: «El problema del significado en las lenguas primitivas», en OGDEN, C.K.; RICHARDS, I.A.: (1910-1922): El significado del significado, Buenos Aires, Paidós, 1964.

MandeVILle, B. de (1723): The Fable of the Bees: or, Private Vices, Publick Benefits, London, Bible and Crown.

MARINA, J. A. (2007): Las arquitecturas del deseo, Barcelona, Anagrama.

MARINAS, J. M.: «Simmel y la cultura del consumo», Reis, 89, 2000.

MARINETTI, F. T. (1929): Primer Manifiesto: «Contra el lujo femenino», Revista de Occidente, 318, 159-163, 2007.

MAuss, M. (1968): «Ensayo sobre los dones», Sociología y Antropología, Madrid, Tecnos, 1991.

MEAdows, D. H. et al. (1972): Los límites del crecimiento, México, Fondo de Cultura Económica, 1982.

Mills. Ch. W. (1963): «The man in the middle: The Designer», Power, Politics and People. The Collected Essays of C. Wright Mills, New York, Oxford University Press. Publicado anteriormente en Industrial Design, nov.1958.

MORACE, F. (1990): Contratendencias. Una nueva cultura del consumo, Madrid, Experimenta, 1993.

PAPANEK, V. (1971): Design for the Real World: Human Ecology and Social Change, Londres, Thames and Hudson, 1985.

PAStOR, A. (2008): «Una ciencia contra la desigualdad», El País, Babelia, 23/02/.

- (2007): La ciencia humilde. Economía para ciudadanos, Barcelona, Crítica.

PRAHADAD, C. K. (2004): La fortuna en la base de la pirámide: cómo crear una vida digna y aumentar las opciones mediante el mercado, Barcelona, Granica, 2005.

REMAURY, B. (2007): «El objeto de lujo en la era de las reproductibilidad técnica». Revista de Occidente, 318, 78-94.

RitZER, G. (1999): El encanto de un mundo desencantado. Revolución en los medios de consumo, Barcelona, Ariel, 2000. 
Romanelli, M. (2006): «Lujo y arquitectura. Vademecum en seis puntos», en Morozzi, C. (ed.): Axolute. Style tech, catálogo de bTicino.

SIMMEL, G. (1917): Cuestiones fundamentales de sociología, Barcelona, Gedisa, 2002.

- (1900): La filosofia del dinero, Instituto de Estudios Políticos, Madrid, 1976.

SLOTERDIJK, P. : La Vanguardia, 3/5/2007.

SoldEVILlA, C. (2001): «Triálogo. Aproximaciones teóricas a la sociología del consumo», Cuadernos de Realidades Sociales, 57-58, Madrid.

SOMBART, W. (1912): Lujo y capitalismo, Revista de Occidente, Madrid, 1928.

VeBLEN, T. (1899): Teoría de la clase ociosa, México, Fondo de Cultura Económica, 1971.

WEBER, M.: Ensayos de sociología contemporánea, Barcelona, Planeta Agostini, 1985.

- (1903): La ética protestante y el espíritu del capitalismo, Barcelona, Península, 1994.

Recibido: 13 de octubre de 2010

Aceptado: 7 de junio de 2011 